\title{
Developing and Implementing a Unified Imaging and Lab Information Management WorkFlow System - Lessons Learned and Insights Gained.
}

\author{
Christopher M. Yip* \\ *Departments of Chemical Engineering and Applied Chemistry, and Biochemistry, Institute of \\ Biomaterials and Biomedical Engineering. University of Toronto 407 - 4 Taddle Creek Rd \\ Toronto, Ontario, Canada M5S 3G9
}

The Centre for Studies in Molecular Imaging (CSMI) is a Canada Foundation for Innovation funded research enterprise located at the University of Toronto (http://bigten.ibme.utoronto.ca/CSMI). A small part of a large research enterprise in cellular and biomolecular research, the CSMI's research mandate is the active promotion of the application of functional molecular-scale imaging tools and techniques, such as confocal and scanning probe microscopy, to soft materials, and in particular, the development of new integrated systems. The unique instrumentation and expertise held within the CSMI, and its functional linkages with other University of Toronto and University Health Network research groups has led to extensive private and non-academic research collaborations. The CSMI provides both support and training facilities for teaching and research and is a showcase facility for its private sector sponsors.

Since its inception, the diverse nature of the data collected by CSMI users, which can range from epifluorescence and confocal images to scanning probe microscopy and force spectroscopy files, computational simulations, and spectroscopic data, has made it difficult to provide a systematic unified file format. Users are often faced with having to migrate between individual workstations in order to analyse their data while off-site users are hampered by not having access to the actual acquisition and analysis software. The large number of instruments, varied skill level of the user base (high school, undergraduate, graduate, professional), and a desire to accommodate the different needs of the users made it necessary to develop a unified imaging and lab information management system. The ideal system would be one that is platform-agnostic, networked, accessible from remote user locations, and secure. A workflow approach was felt to be the most appropriate with all data stored centrally but accessible from all workstations and through secure servers, over the World Wide Web. For our purposes, the ideal system would be one that incorporates a common software package that would allow the user to simultaneous compare data acquired from different instrument in an open architecture.

We will discuss our initial approaches to addressing these needs, which ranged from the implementation of secure FTP servers and dedicated back-up systems, to our most recent projects which bring together Java-enabling technologies, automated user booking and accounting systems, and custom data analysis routines. 


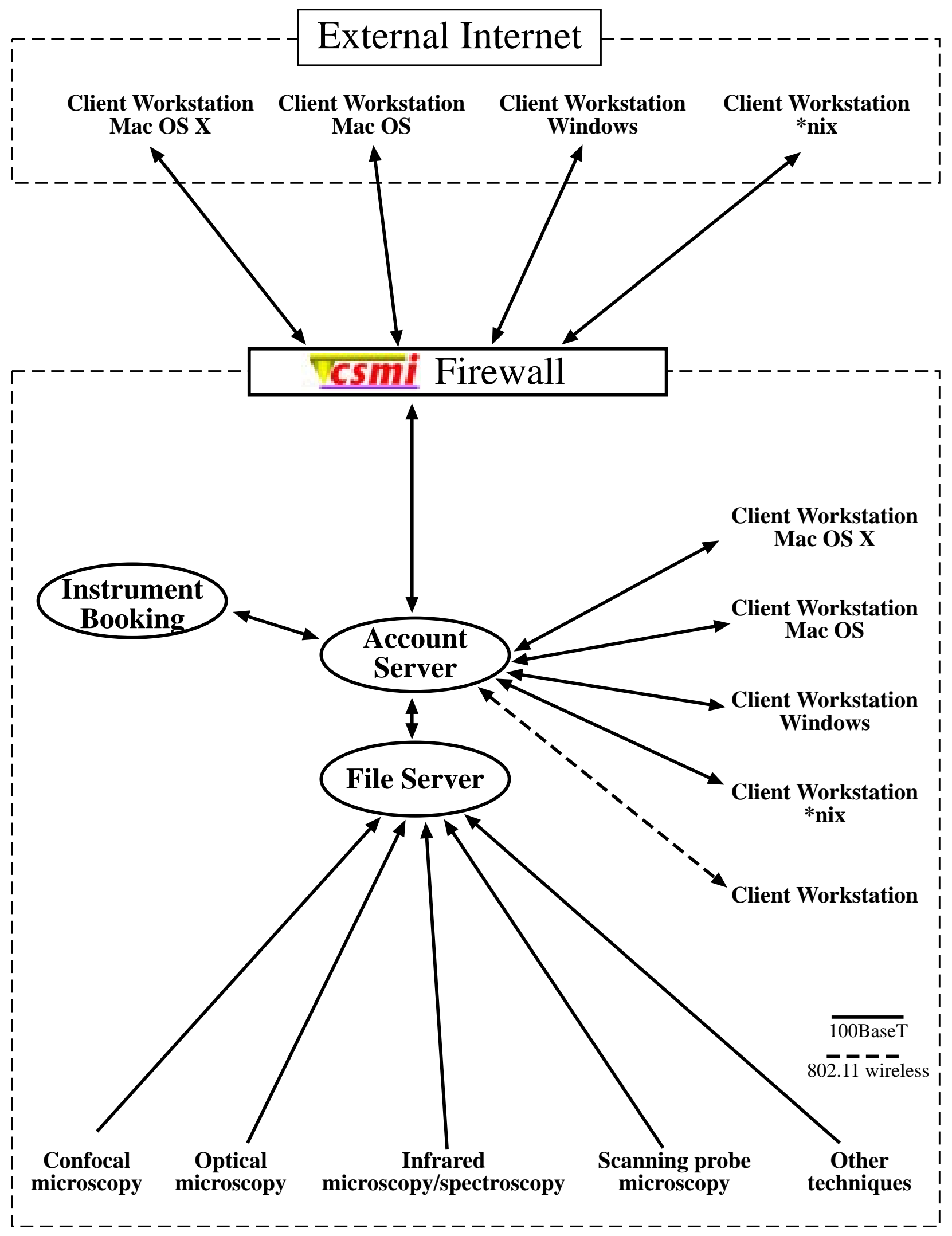

Figure 1. Schematic of CSMI instrumentation and image analysis workflow 